\title{
The Anrep effect requires transactivation of the epidermal growth factor receptor
}

\author{
María C. Villa-Abrille, Claudia I. Caldiz, Irene L. Ennis, Mariela B. Nolly, María J. Casarini, \\ Gladys E. Chiappe de Cingolani, Horacio E. Cingolani and Néstor G. Pérez \\ Centro de Investigaciones Cardiovasculares, Facultad de Ciencias Médicas, Universidad Nacional de La Plata, Calle 60 y 120, 1900 La Plata, Argentina
}

\begin{abstract}
Myocardial stretch elicits a biphasic contractile response: the Frank-Starling mechanism followed by the slow force response (SFR) or Anrep effect. In this study we hypothesized that the SFR depends on epidermal growth factor receptor (EGFR) transactivation after the myocardial stretch-induced angiotensin II (Ang II)/endothelin (ET) release. Experiments were performed in isolated cat papillary muscles stretched from 92 to $98 \%$ of the length at which maximal twitch force was developed $\left(L_{\max }\right)$. The SFR was $123 \pm 1 \%$ of the immediate rapid phase $(n=6, P<0.05)$ and was blunted by preventing EGFR transactivation with the Src-kinase inhibitor PP1 $(99 \pm 2 \%, n=4)$, matrix metalloproteinase inhibitor MMPI $(108 \pm 4 \%, n=11)$, the EGFR blocker AG1478 $(98 \pm 2 \%, n=6)$ or the mitochondrial transition pore blocker clyclosporine $(99 \pm 3 \%, n=6)$. Stretch increased ERK1/2 phosphorylation by $196 \pm 17 \%$ of control $(n=7, P<0.05)$, an effect that was prevented by PP1 $(124 \pm 22 \%, n=7)$ and AG1478 $(131 \pm 17 \%, n=4)$. In myocardial slices, Ang II (which enhances ET mRNA) or endothelin-1 (ET-1)-induced increase in $\mathrm{O}_{2}{ }^{-}$production $(146 \pm 14 \%, n=9$, and $191 \pm 17 \%, n=13$, of control, respectively, $P<0.05)$ was cancelled by AG1478 $(94 \pm 5 \%, n=12$, and $98 \pm 15 \%, n=8$, respectively) or PP1 (100 $\pm 4 \%, n=6$, and $99 \pm 8 \%, n=3$, respectively). EGF increased $\mathrm{O}_{2}{ }^{-}$ production by $149 \pm 4 \%$ of control $(n=9, P<0.05)$, an effect cancelled by inhibiting NADPH oxidase with apocynin $(110 \pm 6 \% n=7)$, mKATP channels with 5-hydroxydecanoic acid (5-HD; $105 \pm 5 \%, n=8)$, the respiratory chain with rotenone $(110 \pm 7 \%, n=7)$ or the mitochondrial permeability transition pore with cyclosporine $(111 \pm 10 \%, n=6)$. EGF increased ERK1/2 phosphorylation ( $136 \pm 8 \%$ of control, $n=9, P<0.05)$, which was blunted by $5-\mathrm{HD}(97 \pm 5 \%$, $n=4)$, suggesting that ERK1/2 activation is downstream of mitochondrial oxidative stress. Finally, stretch increased Ser703 Na$/ / \mathrm{H}^{+}$exchanger-1 (NHE-1) phosphorylation by $172 \pm 24 \%$ of control $(n=4, P<0.05)$, an effect that was cancelled by AG1478 $(94 \pm 17 \%, n=4)$. In conclusion, our data show for the first time that EGFR transactivation is crucial in the chain of events leading to the Anrep effect.
\end{abstract}

\begin{abstract}
(Received 22 December 2009; accepted after revision 9 March 2010; first published online 15 March 2010) Corresponding author N. G. Pérez: Centro de Investigaciones Cardiovasculares, Facultad de Ciencias Médicas, UNLP, 60 y 120 (1900) La Plata, Argentina. Email: gperez@med.unlp.edu.ar

Abbreviations Ang II, angiotensin II; ET, endothelin; EGF, epidermal growth factor; EGFR, epidermal growth factor receptor; GPCR, G protein coupled receptor; 5-HD, 5-hydroxydecanoic acid; $L_{\max }$, the length at which maximal twitch force was developed; MMP, matrix metalloproteinase; MMPI, MMP inhibitor; MPTP, mitochondrial permeability transition pore; NCX, Na ${ }^{+} / \mathrm{Ca}^{2+}$ exchanger; NHE-1, $\mathrm{Na}^{+} / \mathrm{H}^{+}$exchanger-1; $\mathrm{O}_{2}^{-}$, superoxide anion; PP1, Src-kinase inhibitor; ROS, reactive oxygen species; SFR, slow force response.
\end{abstract}

\section{Introduction}

In myocytes, angiotensin II (Ang II) is stored in secretory vesicles, and its release is triggered by mechanical stretch (Sadoshima et al. 1993; Leri et al. 1998). The release of Ang II is sufficient to activate angiotensin 1 (AT1) receptors in an autocrine/paracrine loop that leads to the release/formation of endothelin (ET) (Ito et al. 1993; Yamazaki et al. 1996, 1998), the production of NADPH-dependent mitochondrial reactive oxygen species (ROS) (Zhou et al. 2006; Caldiz et al. 2007; Zhang et al. 2009), the activation of redox sensitive kinases, and the stimulation of the $\mathrm{Na}^{+} / \mathrm{H}^{+}$exchanger-1 (NHE-1) (Akram et al. 2006; Caldiz et al. 2007). One consequence of this chain of events appears to be myocardial hypertrophy (Ennis et al. 2007). 
In concert with the autocrine/paracrine loop, myocardial stretch triggers an increase in cardiac contractility that develops immediately after the Frank-Starling mechanism, reaching a maximum after 10 to $15 \mathrm{~min}$. This is due to an increase in the intracellular calcium transient, without changes in myofilament calcium sensitivity (Allen \& Kurihara, 1982; Kentish \& Wrzosek, 1998; Perez et al. 2001). This mechanical counterpart of the autocrine/paracrine loop is called the slow force response to stretch (SFR) (Parmley \& Chuck, 1973; Calaghan \& White, 2001) or the Anrep effect (von Anrep, 1912; Sarnoff et al. 1960; Alvarez et al. 1999). The SFR seems to involve the early intracellular signals that lead to myocardial hypertrophy; in particular, mitochondrial ROS formation seems to be crucial (Caldiz et al. 2007; Zhang et al. 2009). Interestingly, recent evidence suggested that Ang II, the first step in the chain of events that leads to SFR, requires the transactivation of endothelial growth factor receptor (EGFR) to induce cardiac hypertrophy (Kagiyama et al. 2002; Zhai et al. 2006; Szokodi et al. 2008). Furthermore, in neonatal cardiac myocytes, stretch elicited a mechanism that required EGFR transactivation to induce the release/formation of ET and, consequently, the activation of extracellular signal-regulated kinase (ERK) (Asakura et al. 2002; Duquesnes et al. 2009). We therefore hypothesised that transactivation of the EGFR must be critical for the development of the SFR.

This study investigated whether blunting the $G$ protein coupled receptor (GPCR)-mediated transactivation of EGFR would block the SFR. A preliminary report of our data has been presented elsewhere (Perez et al. 2009).

\section{Methods}

All procedures followed during this investigation conform to the Guide for the Care and Use of Laboratory Animals published by the US National Institutes of Health (NIH Publication No. 85-23, revised 1996), to the guidelines laid down by the Animal Welfare Committee of the La Plata School of Medicine, and the policies of The Journal of Physiology (Drummond, 2009). Cats $(n=42$, body weight $3-4 \mathrm{~kg}$ ) were anaesthetized by intraperitoneal injection of sodium pentobarbitone $(35 \mathrm{mg}$ ( $\mathrm{kg}$ body weight $)^{-1}$ ). The chests were opened to excise the heart when deep anaesthesia was reached, verified by the loss of corneal reflex and appearance of slow deep diaphragmatic breathing. Animals were provided by a local supplier (San Cayetano, Monte Grande, Argentina). Each heart was used to provide papillary muscles as well as cardiac tissue slices.

\section{Isolation of papillary muscles}

Papillary muscles from the right ventricle were used to assess the SFR to stretch as previously described
(Perez et al. 2001). Briefly, the muscles were mounted in a perfusion chamber placed on the stage of an inverted microscope (Olympus) and superfused at a constant rate $\left(5 \mathrm{ml} \mathrm{min}{ }^{-1}\right)$ with a $\mathrm{CO}_{2} / \mathrm{HCO}_{3}{ }^{-}$-buffered solution containing $\left(\mathrm{mmoll}^{-1}\right): \mathrm{NaCl} 128.3, \mathrm{KCl} 4.5$, $\mathrm{CaCl}_{2}$ 1.35, $\mathrm{NaHCO}_{3}$ 20.23, $\mathrm{MgSO}_{4} 1.05$, glucose 11.0 and equilibrated with $5 \% \mathrm{CO}_{2}-95 \% \mathrm{O}_{2}(\mathrm{pH} \sim 7.40)$. The possible participation of catecholamines released by the nerve endings was prevented by adrenergic receptors blockade with $1.0 \mu \mathrm{moll}^{-1}$ prazosin plus $1.0 \mu \mathrm{moll}^{-1}$ atenolol. The muscles were paced at $0.2 \mathrm{~Hz}$ at a voltage $10 \%$ over threshold maintained at $30^{\circ} \mathrm{C}$, and isometric contractions were recorded. Cross sectional area (calculated as 0.75 of the product of thickness and width) was used to normalize force records obtained with a silicon strain gauge (model AEM 801, Sensonor Technologies, Horten, Norway). The slack length of each muscle was determined after mounting, and then the muscles were progressively stretched to the length at which they developed maximal twitch force $\left(L_{\max }\right)$. After a few minutes at $L_{\max }$, they were shortened to obtain the $95 \%$ of the maximal twitch force (length that approximated $98 \%$ of $L_{\max }$ and referred to as $\left.L_{98}\right)$. Then, the muscles were shortened to $92 \%$ of $L_{\max }$ $\left(L_{92}\right)$ and maintained at this length until the beginning of the experimental protocol, when they were abruptly stretched from $L_{92}$ to $L_{98}$. The drugs were added to the perfusate $20 \mathrm{~min}$ before stretch, and during this period, none of them changed the developed force by more than $3 \%$ to $4 \%$.

\section{Cardiac tissue slices}

Preparation of cardiac slices. Hearts were quickly removed from previously anaesthetized animals. The hearts were perfused through the aorta with Krebs-Ringer buffer $\mathrm{pH} 7.4$ to eliminate the blood, and tissue slices from the left ventricle $(1 \times 5 \mathrm{~mm})$ were cut and kept at $4^{\circ} \mathrm{C}$ until assayed. Assay buffer consisted of Krebs-Hepes buffer of the following composition (in $\mathrm{mmol}^{-1}$ ): $118.3 \mathrm{NaCl} ; 4.7$ $\mathrm{KCl} ; 1.8 \mathrm{CaCl}_{2} ; 1.2 \mathrm{MgSO}_{4} ; 1.0 \mathrm{~K}_{2} \mathrm{HPO}_{4} ; 25 \mathrm{NaHCO}_{3} ; 11$ glucose; 20 Hepes (pH 7.4 after $1.5 \mathrm{~h}$ aeration with $95 \%$ $\mathrm{O}_{2}-5 \% \mathrm{CO}_{2}$ at $\left.37^{\circ} \mathrm{C}\right)$. Cardiac tissues were incubated in assay buffer during $30 \mathrm{~min}$ in the presence of different drugs in a metabolic incubator under $95 \% \mathrm{O}_{2}-5 \% \mathrm{CO}_{2}$ at $37^{\circ} \mathrm{C}$ before measurements of $\mathrm{O}_{2}^{-}$production.

\section{Measurements of superoxide anions $\left(\cdot \mathrm{O}_{2}^{-}\right)$production.} We used lucigenin-enhanced chemiluminescence to measure $\mathrm{O}_{2}{ }^{-}$production by cat cardiac tissue in Krebs-Hepes buffer with $5 \mu \mathrm{mol} \mathrm{l}^{-1}$ lucigenin as described previously (Caldiz et al. 2007). The chemiluminescence in arbitrary units (AU) was recorded with a luminometer (Chameleon; Hidex, Turku, Finland) during $30 \mathrm{~s}$, each with a $4.5 \mathrm{~min}$ interval during $30 \mathrm{~min} . \mathrm{O}_{2}{ }^{-}$production was 
normalized to milligrams dry weight tissue per minute. Control tissue slices without any pharmacological intervention produced low levels of $\mathrm{O}_{2}{ }^{-}$that were only slightly above background. Although the lucigenin-enhanced chemiluminescence method does not allow accurate detection of the basal $\mathrm{O}_{2}{ }^{-}$production (Dikalov et al. 2007), its sensitivity was enough to detect an increase in $\mathrm{O}_{2}{ }^{-}$production above background after Ang II, ET- 1 and EGF. When necessary, the increase in $\mathrm{O}_{2}{ }^{-}$production was expressed as a percentage of basal value after $15 \mathrm{~min}$ of each intervention.

\section{Determination of extracellular signal-regulated protein kinases (ERK1/2) and NHE-1 phosphorylation}

Cardiac tissue was homogenized in lysis buffer (300 $\mathrm{mmoll}^{-1}$ sucrose; $1 \mathrm{mmol}^{-1}$ dithiothreitol (DTT); $4 \mathrm{mmol}^{-1}$ EGTA, protease inhibitor cocktail (Complete Mini, Roche); $20 \mathrm{mmol}^{-1}$ Tris-HCl, pH 7.4). After a brief centrifugation the supernatant was kept and protein concentration determined by the Bradford method. Samples were denatured and equal amounts of protein were subjected to PAGE and electrotransferred to PVDF membranes. Membranes were then blocked with non-fat dry milk and incubated overnight with either anti-phospho-ERK1/2 or anti-ERK 2 (Santa Cruz Biotechnology, Santa Cruz, CA, USA). For NHE-1 phosphorylation determination, samples were immunoprecipitated using a NHE-1 polyclonal antibody (Chemicon, Temecula, CA, USA) and then subjected to PAGE, electrotransferred and incubated with an anti-14-3-3 binding motif antibody (Cell Signaling Technology, Inc., Danvers, MA, USA). Previous reports have shown that the regulatory Ser703 of the NHE-1 lies within a sequence which creates upon phosphorylation a binding motif for 14-3-3 proteins (Takahashi et al. 1999; Lehoux et al. 2001). Thus, the anti-P-14-3-3 binding motif antibody when probed with immunoprecipitated NHE-1 represents a useful tool to estimate NHE-1 phosphorylation at Ser703 (Snabaitis et al. 2006). Total ERK-2 and NHE-1 as a loading control were assayed. Peroxidase-conjugated anti-rabbit or anti-mouse IgG (Santa Cruz Biotechnology) was used as secondary antibody and bands were visualized using the ECL-Plus chemiluminescence detection system (GE Healthcare, Little Chalfont, UK). Autoradiograms were analysed by densitometric analysis (Scion Image).

\section{RNA measurements by RT-PCR}

Total RNA was isolated from the cardiomyocytes suspension using the RNeasy kit (Qiagen, Valencia, CA, USA) according to the manufacturer's instructions. RNA $(0.8 \mu \mathrm{g})$ was reverse-transcribed using the Omniscript RT kit (Qiagen). A dilution of the resulting cDNA was used for quantifying the relative content of mRNA by real-time PCR (iCycler iQ Real-Time PCR Detection System, Bio-Rad) using appropriate primers and SYBR Green as a fluorescent probe. The following primers, designed using Primer3 software, were used: GAPDH: forward primer 5-GGGTGTGAACCACGAGAAAT-3; reverse primer 5-CCACAGTCTTCTGAGTGGCA-3; preproET-1: forward primer 5-CAGACAAAGAACTCCGAGCC-3; reverse primer 5-GGTCTTGATGCTGTTGCTGA-3. PCR reactions were performed with TaqDNA polymerase (Invitrogen). Fluorescence data were acquired at the end of extension. A melt analysis was run for all of the products to determine the specificity of the amplification. The cycle threshold values for each gene were measured and calculated by computer software (iCycler IQ OSS, version 3.0a, Bio-Rad). The cycle threshold values for each gene were normalized by those GAPDH in the same sample. The primers were designed against the feline sequences. For each experiment of real-time RT-PCR we obtained a melting curve of the amplified gene where it could be appreciated that only a specific product was amplified in each case with no primer-dimer formation.

\section{Statistics}

Results are expressed as means \pm S.E.M. Differences between initial phase and post-stretch records, comparison of $\mathrm{O}_{2}{ }^{-}$production after 15 min of incubation with the different drugs and ERK1/2 phosphorylation under the different conditions were assessed by one-way ANOVA followed by the Student-Newman-Keuls test. Two-way ANOVA was used to compare post-stretch data in the absence and presence of drugs. $P<0.05$ was considered significant.

\section{Results}

\section{Probing the intracellular signalling pathway triggered by myocardial stretch}

Figure $1 A$ shows a typical force response to stretch when an isolated cat papillary muscle is stretched from 92 to $98 \%$ of its maximum length. The result is a biphasic contractile response: the classical Frank-Starling mechanism is manifested immediately after stretch, followed by a gradually developing increase in force, known as the SFR to stretch or the Anrep effect.

Previous studies from our and other laboratories have provided evidence that the SFR is the result of an autocrine/paracrine loop that begins with the activation of GPCRs (Ang II and ET) and ends with an increase in the $\mathrm{Ca}^{2+}$ transient through the $\mathrm{Na}^{+} / \mathrm{Ca}^{2+}$ exchanger (Cingolani et al. 1998; Yamazaki et al. 1998; Alvarez et al. 
1999; Calaghan \& White, 2001; Perez et al. 2001, 2003; Luers et al. 2005). The role of mitochondrial ROS in the intracellular signalling pathway was also recently described (Caldiz et al. 2007; Zhang et al. 2009).

In order to probe the connection between EGFR transactivation and the SFR, we explored whether inhibiting EGFR transactivation would affect the SFR of a papillary muscle. Several mediators are known to be involved in the transactivation process, but the precise communication between the GPCR and EGFR is not entirely understood (Wetzker \& Bohmer, 2003). One proposed mediator is Src tyrosine kinase (Wetzker \& Bohmer, 2003); thus, as a first test of our hypothesis, we inhibited Src kinase with the specific tyrosine kinase inhibitor PP1. Figure $1 C$ shows that PP1 $\left(1 \mu \mathrm{moll}^{-1}\right)$ completely cancelled the SFR to stretch. Another proposed mediator (Krieg et al. 2004; Szokodi et al. 2008) of EGFR transactivation was heparin-binding EGF (HB-EGF). HB-EGF is generated through extracellular proteolytic cleavage of proHB-EGF by the action of a matrix metalloproteinase (MMP). To test the contribution of this signalling pathway to the SFR, we inhibited MMP with MMP inhibitor III (MMPI), which specifically targeted MMPs $1,2,3,7$ and 13. MMPI $\left(3 \mu \mathrm{mol}^{-1}\right)$ did not completely eliminate the SFR, but significantly reduced its magnitude by $\sim 60 \%$ (Fig. 1B). This provided further support of the notion that EGFR transactivation was required for a fully developed SFR.

Finally, we specifically inhibited the EGFR with AG1478 $\left(1 \mu \mathrm{moll}^{-1}\right)$, which is known to prevent receptor phosphorylation, and consequently, blocks receptor activation. Under these conditions, the SFR was completely abolished (Fig. 1D). The average SFR observed under the different experimental conditions was expressed as a percentage of the initial rapid phase (Fig. 1E).

Thus, these three interventions that interfered with the mechanism of transactivation significantly decreased the SFR. These results support the hypothesis that EGFR transactivation plays a role in the development of the SFR in cat myocardium.

\section{Activation of redox sensitive kinases after myocardial stretch}

We (Cingolani et al. 2005; Caldiz et al. 2007) and others (Zhang et al. 2009) have previously shown that the SFR depends on the activation of NHE-1, which is a target of the redox sensitive kinases, ERK1/2. Others showed that ROS stimulated NHE-1 through MAPK (Rothstein et al. 2002; Haworth et al. 2003; Fliegel \& Karmazyn, 2004; Akram et al. 2006), and we recently proposed that stretch induced the mitochondrial production of ROS (Caldiz et al. 2007).

In this study, muscles were frozen 5 min after myocardial stretch and processed for kinase detection. We detected a significant increase in ERK1/2 phosphorylation. This effect was cancelled by pre-treatment with either AG1478 or PP1 (Fig. 2), two inhibitors of EGFR transactivation that also blocked the mechanical response. These findings showed that the prevention of EGFR phosphorylation and activation is able to cancel both the increase in ERK1/2 phosphorylation and the mechanical response to stretch.
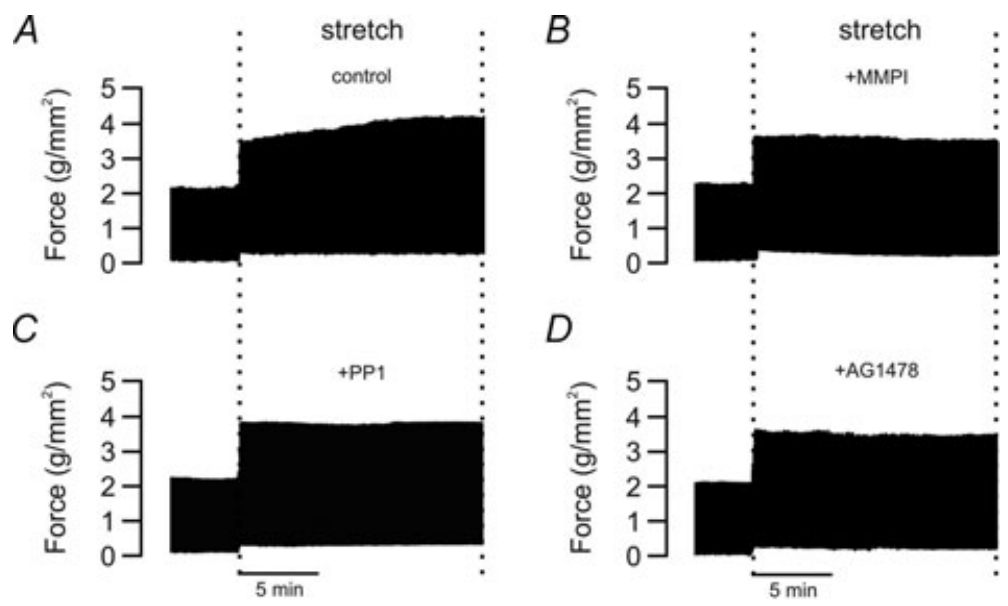

E

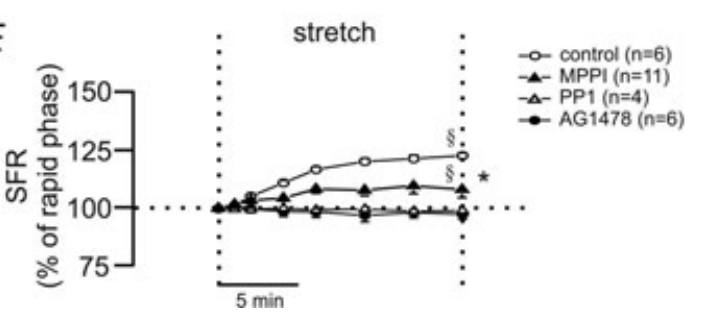

Figure 1. SFR and EGFR transactivation $A$, a typical force record from a cat papillary muscle subjected to an increase in length from 92 to $98 \%$ of $L_{\text {max }}$; the biphasic response to stretch can be seen (vertical dotted lines indicate stretching interval). $B-D$, same as $A$ but from muscles pretreated with matrix metalloproteinase inhibitor (MMPI, $B$ ), the Src kinase inhibitor PP1 (C) or the EGFR blocker AG1478 (D), interventions that cancel EGFR transactivation. As can be seen, all these pharmacological interventions prevented the development of the SFR to stretch. $E$, the averaged results obtained under the different experimental conditions expressed as a percentage of the initial rapid phase. ${ }^{*} P<0.05$ control curve vs. others ( 2 -way ANOVA). $\S P<0.05$ vs. initial rapid phase (for the sake of clarity, significance is indicated only for 15 min of stretch). 


\section{Ang II, ET-1, ROS production and transactivation of the EGFR}

We proposed that mitochondrial ROS are a necessary upstream signal for ERK1/2 activation, NHE-1 phosphorylation, and SFR development. Our proposal was based on evidence that physiological concentrations of exogenous Ang II or ET-1 induced a NADPH-dependent increase in $\mathrm{O}_{2}{ }^{-}$formation, and this could be blunted by interfering with the mitochondrial ROS source (Caldiz et al. 2007). Here, we found that either AG1478 or PP1 suppressed Ang II/ET-1-induced $\mathrm{O}_{2}{ }^{-}$formation in cat myocardial slices (Fig. 3). These results implied that EGFR transactivation is critical in mediating the Ang II and ET-1 induction of ROS production.

\section{Ang II-ET-1 cross talk}

Myocardial stretch causes the release of preformed Ang II from neonatal (Sadoshima et al. 1993) and adult myocytes (Leri et al. 1998). The effects induced by physiological Ang II concentrations can be blunted by interfering with ET-1, either by blocking the endothelin type-A (ETA) receptor or by inhibiting the ET conversion enzyme (Alvarez et al. 1999; Perez et al. 2001; Cingolani et al. 2005, 2006). Furthermore, the presence of prepro-ET, Big ET, and ET was detected in the cardiomyocytes (Cingolani et al. 2006). Therefore, myocardial stretch induces Ang II and/or ET actions.

$A$
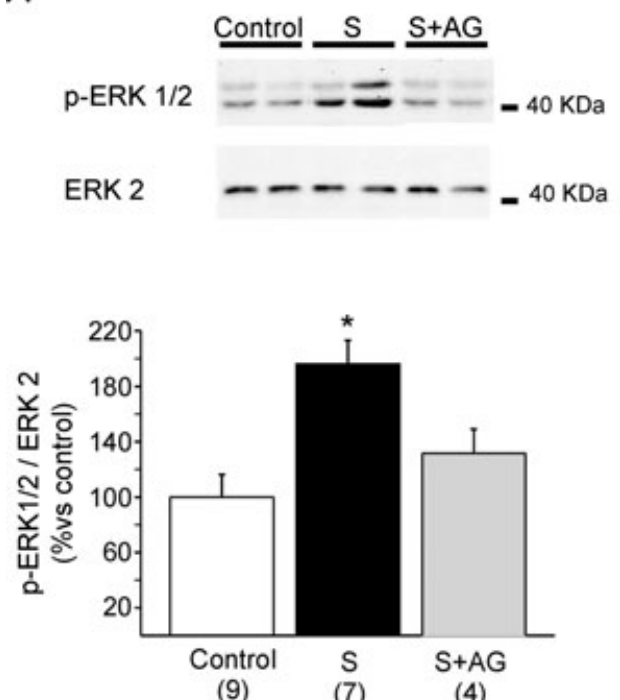

(9)

(4)
The mechanism by which Ang II elicited ET release/formation is not fully known. Based on studies on B-type natriuretic peptide production in neonates, Anderson et al. (2004) proposed that stretch induced ET release through a NADPH-dependent, redox-sensitive mechanism. Consistent with his proposal, we found that $1 \mathrm{nmoll}^{-1}$ of Ang II increased the mRNA expression of ET-1 in cat myocytes (Fig. 4), perhaps reflecting the replenishment of a released pool and/or increased ET-1 formation. This effect was inhibited by the AT1 blocker losartan, the NADPH inhibitor apocynin and the ROS scavenger 2-mercaptopropionyl glycine. The exact mechanism of the cross talk between Ang II and ET-1 is out of the scope of this study; however, these findings showed that the cross talk between Ang II and ET-1 was sensitive to the redox state in the adult feline heart.

\section{Increased ROS formation by EGF signalling}

In order to investigate this mechanism further, we evaluated whether EGF was able to increase $\mathrm{O}_{2}{ }^{-}$formation under our experimental conditions. We found that EGF increased myocardial $\mathrm{O}_{2}{ }^{-}$production in a dose-dependent manner (Fig. 5). The effect of EGF at $0.1 \mu \mathrm{g} \mathrm{ml}^{-1}$ was similar to the increase in $\mathrm{O}_{2}^{-}$production observed with $1 \mathrm{nmol}^{-1}$ ET-1; thus, we decided to use this EGF concentration in the following experiments. The increase in $\mathrm{O}_{2}{ }^{-}$production induced by $0.1 \mu \mathrm{g} \mathrm{ml}^{-1} \mathrm{EGF}$ was, as expected, cancelled by pre-treatment with the EGFR

\section{$B$}

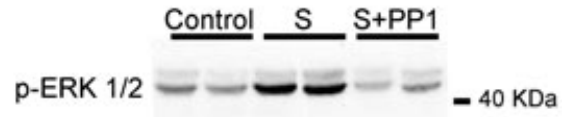

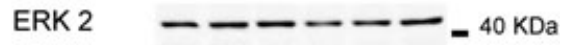

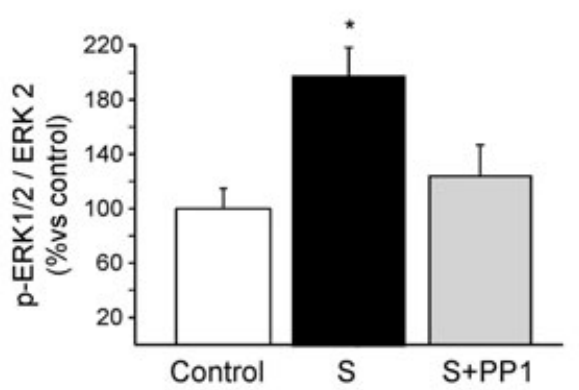

(8)
(7)

Figure 2. ERK1/2 phosphorylation after stretch Five minutes of stretch significantly increased ERK1/2 phosphorylation, kinases upstream NHE-1, that we have previously shown to play a critical role in SFR development (Caldiz et al. 2007). This effect was blunted either by EGFR blockade with AG1478 (AG, A) or by Src kinase inhibition with PP1 (B), demonstrating that EGFR transactivation after stretch is necessary for ERK1/2 phosphorylation. It is important to highlight that either AG1478 or PP1 alone did not modify basal ERK1/2 phosphorylation $(92 \pm 4 \%, n=4$, and $107 \pm 8 \%, n=4$, of control respectively). ${ }^{*} P<0.05$ vs. non-stretched control. 
blocker AG1478, but not by the Src kinase inhibitor PP1 (Fig. $5 B$ ), which is upstream of the EGFR transactivation pathway (Wetzker \& Bohmer, 2003; Krieg et al. 2004). As reported previously for Ang II/ET-1 (Caldiz et al. 2007), the increase in ROS production was NADPH dependent, since it was eliminated by apocynin (Fig. 5B). Furthermore, EGF-induced ROS production was abrogated by blocking mitochondrial ATP-sensitive potassium channels (mKATP) with 5-hydroxydecanoic acid (5-HD; a mKATP-specific blocker) and glybenclamide (a non-selective KATP blocker), or the mitochondrial respiration inhibitor rotenone (Fig. $5 B$ ). Thus, these data support the notion that Ang II elicits an increase in mitochondrial ROS formation through an intracellular signalling pathway that involves ET and EGFR transactivation.

\section{ERK1/2 phosphorylation by EGF: role of mitochondria}

Next, we reasoned that, if Ang II/ET-1 generated the ROS that activated the ERK1/2 kinases and led to
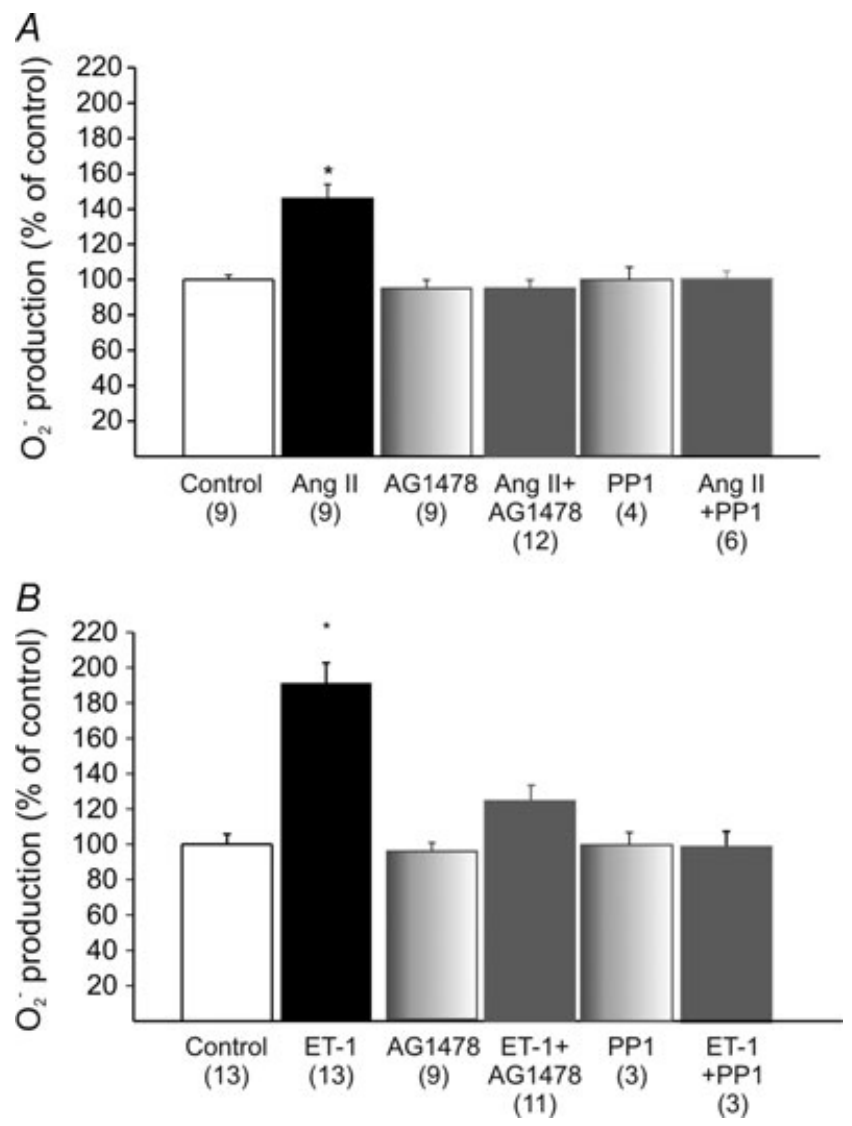

Figure 3. $\mathrm{O}_{2}^{-}$production after addition of Ang II or ET-1 in cardiac slices

The increase in $\mathrm{O}_{2}^{-}$production induced either by $1 \mathrm{nmol} \mathrm{I-1}$ Ang II (A) or $1 \mathrm{nmol} \mathrm{I}^{-1}$ ET-1 (B) was cancelled by AG1478 or PP1. These findings demonstrate that the well known increase in ROS production after Ang II/ET-1 treatment requires EGFR transactivation in the myocardium. ${ }^{*} P<0.05$ vs. control.
NHE-1 phosphorylation (Rothstein et al. 2002; Haworth et al. 2003; Fliegel \& Karmazyn, 2004; Caldiz et al. 2007), and these effects depended on EGFR transactivation, then EGF should be able to induce ERK1/2 phosphorylation. Figure 6 shows that an increase in ERK1/2 phosphorylation was induced by $0.1 \mu \mathrm{g} \mathrm{ml}^{-1}$ EGF. This increase was abolished by AG1478 (not shown), and also by inhibiting the NADPH oxidase (apocynin), the mKATP channels (with 5-HD or glybenclamide) or the mitochondrial respiratory chain (rotenone). This clearly suggested that mitochondrial ROS formation was a necessary step upstream of ERK 1/2 phosphorylation.

\section{Role of mitochondrial permeability transition pore (MPTP) in SFR}

Based on the above data, we could conclude that SFR development depended on EGFR transactivation inducing the formation of mitochondrial ROS. Transactivation may induce mitochondrial ROS formation by promoting the opening of mKATP channels. This would, in turn, modulate MPTP formation and lead to an increase in $\mathrm{O}_{2}{ }^{-}$ production. If the MPTP were involved in mitochondrial ROS release, then the prevention of MPTP opening should prevent ROS formation and thus block the SFR to stretch. Figure 7 shows that this was indeed the case. Pretreatment of papillary muscles with the MPTP inhibitor cyclosporine A $\left(0.5 \mu \mathrm{moll}^{-1}\right)$ completely cancelled the SFR.

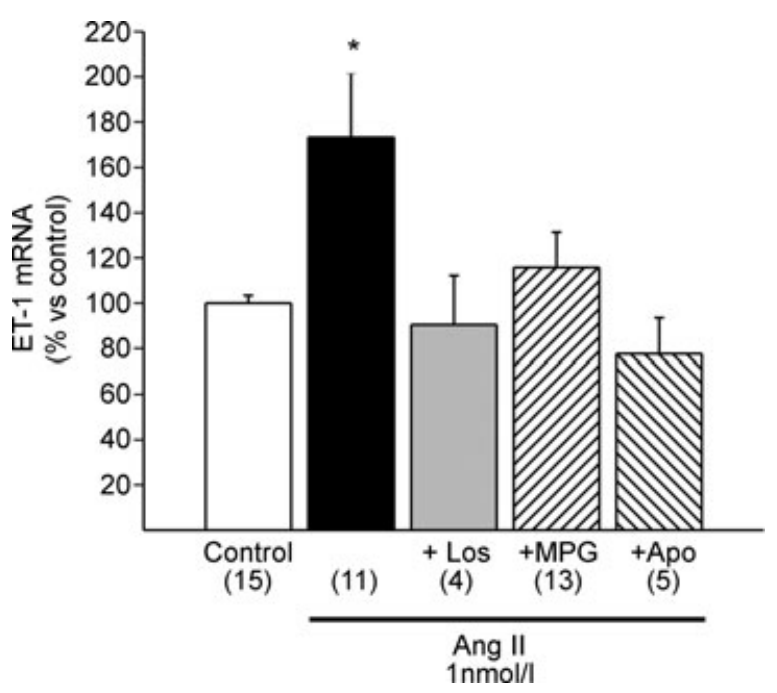

Figure 4. Ang II-ET-1 crosstalk

Ang II (1 nmol I-1) promoted a significant increase in preproET-1 mRNA expression detected by real-time RT-PCR, a result that confirms the Ang II-ET crosstalk in the cat myocardium. This increase in preproET-1 mRNA was, as expected, not only cancelled by AT1 receptor blockade with losartan (LoS), but also by scavenging ROS with 2-mercaptopropionyl glycine (MPG) or by inhibiting NADPH oxidase with apocynin (Apo), indicating that NADPH oxidase-mediated ROS production is a key step to increase ET-1 expression. ${ }^{*} P<0.05$ vs. control. 
$A$

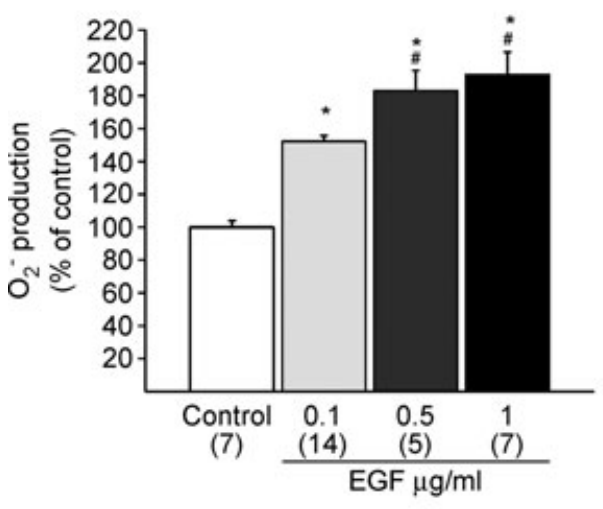

$B$

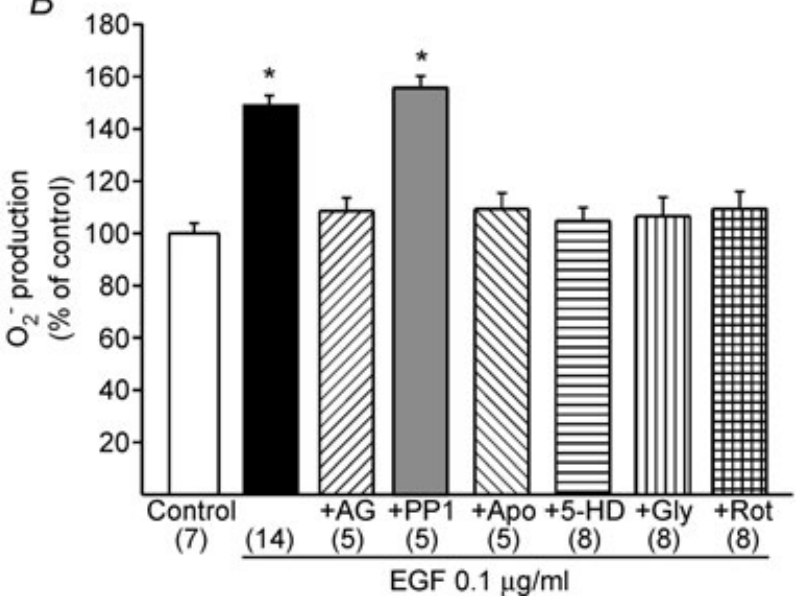

Figure 5. $\mathrm{O}_{2}^{-}$production after addition of EGF in cardiac slices

Direct activation of the EGFR by EGF significantly enhanced $\mathrm{O}_{2}{ }^{-}$production in a dose-dependent manner $(A)$. We selected the lower concentration of EGF $\left(0.1 \mu \mathrm{g} \mathrm{ml}^{-1}\right)$ to perform subsequent experiments because it increased $\mathrm{O}_{2}^{-}$production in a similar magnitude to $1 \mathrm{nmol} \mathrm{I}^{-1} \mathrm{ET}-1$ (see Fig. 3). As expected, the $0.1 \mu \mathrm{g} \mathrm{ml}^{-1} \mathrm{EGF}$ effect was blunted by specific EGFR blockade with AG1478 (AG) but not by Src kinase inhibition with PP1 (B) since the latter is located upstream of EGFR. The EGF-induced increase in $\mathrm{O}_{2}{ }^{-}$production was also prevented by NADPH oxidase inhibition with apocynin (Apo), by two different mitochondrial ATP-sensitive $\mathrm{K}^{+}$channel (mKATP) blockers (5-hydroxydecanoate (5-HD) and glybenclamide (Gly)), and also by the respiratory chain inhibitor rotenone (Rot). These data support the hypothesis that EGF is a necessary mediator in the stretch-induced mitochondrial ROS production. * and \# indicate $P<0.05 \mathrm{vs}$. control and $0.1 \mu \mathrm{g} \mathrm{ml}^{-1}$ EGF respectively.

In addition, cyclosporine prevented the EGF-mediated increase in $\mathrm{O}_{2}{ }^{-}$production (Fig. 7).

The changes in $\mathrm{O}_{2}{ }^{-}$production were measured by the lucigenin method; thus, we could not determine whether the activation of kinases and NHE was mediated by $\mathrm{O}_{2}{ }^{-}$or $\mathrm{H}_{2} \mathrm{O}_{2} \cdot \mathrm{O}_{2}{ }^{-}$is very unstable, and it is rapidly converted by superoxide dismutase (SOD) into $\mathrm{H}_{2} \mathrm{O}_{2}$, a more stable, membrane-permeant ROS that is widely recognized as a signalling molecule. In the myocardium, dismutation is performed by a membrane bound extracellular SOD, a cytoplasmic CuZn SOD, and a mitochondrial Mn SOD. Therefore, the $\mathrm{O}_{2}{ }^{-}$detected in cardiac slices might be easily converted into $\mathrm{H}_{2} \mathrm{O}_{2}$ at different cellular locations, upstream or downstream of the mitochondria. Furthermore, Sabri et al. (1998) and Rothstein et al. (2002) demonstrated that $\mathrm{H}_{2} \mathrm{O}_{2}$ was the intracellular signal that led to the activation of kinases that phosphorylated the NHE; furthermore, we have previously shown that promoting an increased conversion of $\mathrm{O}_{2}{ }^{-}$into $\mathrm{H}_{2} \mathrm{O}_{2}$ led to an enhanced SFR (Caldiz et al. 2007). These results suggested that $\mathrm{H}_{2} \mathrm{O}_{2}$ plays an important role in the development of the SFR or Anrep effect.

\section{NHE-1 phosphorylation}

We (Cingolani et al. 1998; Alvarez et al. 1999; Cingolani et al. 2005) and others (Calaghan \& White, 2001; Luers et al. 2005) have previously demonstrated that the SFR was cancelled by NHE-1 inhibitors in different species. The underlying mechanism was the prevention of the NHE-1 activation-induced increase in intracellular $\mathrm{Na}^{+}$that would favour intracellular $\mathrm{Ca}^{2+}$ accumulation through the
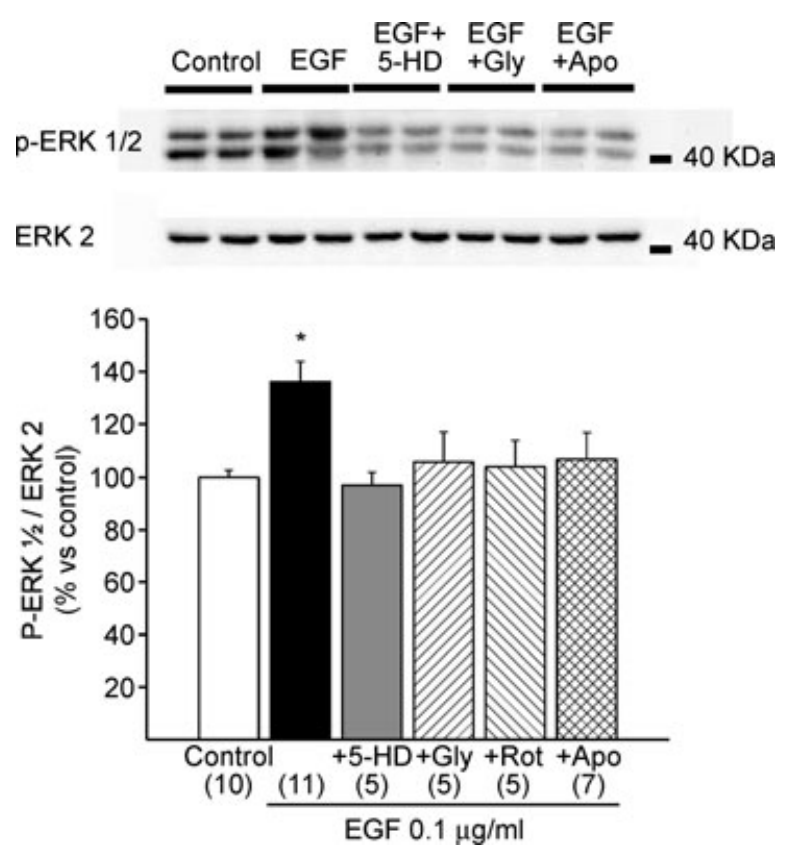

Figure 6. ERK1/2 phosphorylation after EGF stimulation EGF significantly increased ERK $1 / 2$ phosphorylation, an effect that was cancelled by 5-HD, glybenclamide (Gly), rotenone (Rot) or apocynin (Apo). These results further support the notion that mitochondrial ROS formation is a necessary upstream step to ERK $1 / 2$ phosphorylation. $* P<0.05$ vs. control. 
$\mathrm{Na}^{+} / \mathrm{Ca}^{2+}$ exchanger (Alvarez et al. 1999; Perez et al. 2001, 2003).

Previous reports (Takahashi et al. 1999; Lehoux et al. 2001; Snabaitis et al. 2006) have shown that Ser703 in the regulatory carboxyl-tail of NHE-1 creates a binding motif for 14-3-3 proteins upon phosphorylation by ERK1/2-p90RSK cascade. We estimated the levels of NHE-1 phosphorylation at Ser703 with a phospho-Ser 14-3-3 binding motif antibody. Figure 8 shows that phosphorylation in the 14-3-3 binding motif was increased with myocardial stretch and the increase was prevented with AG1478. A limitation of our study is that NHE-1
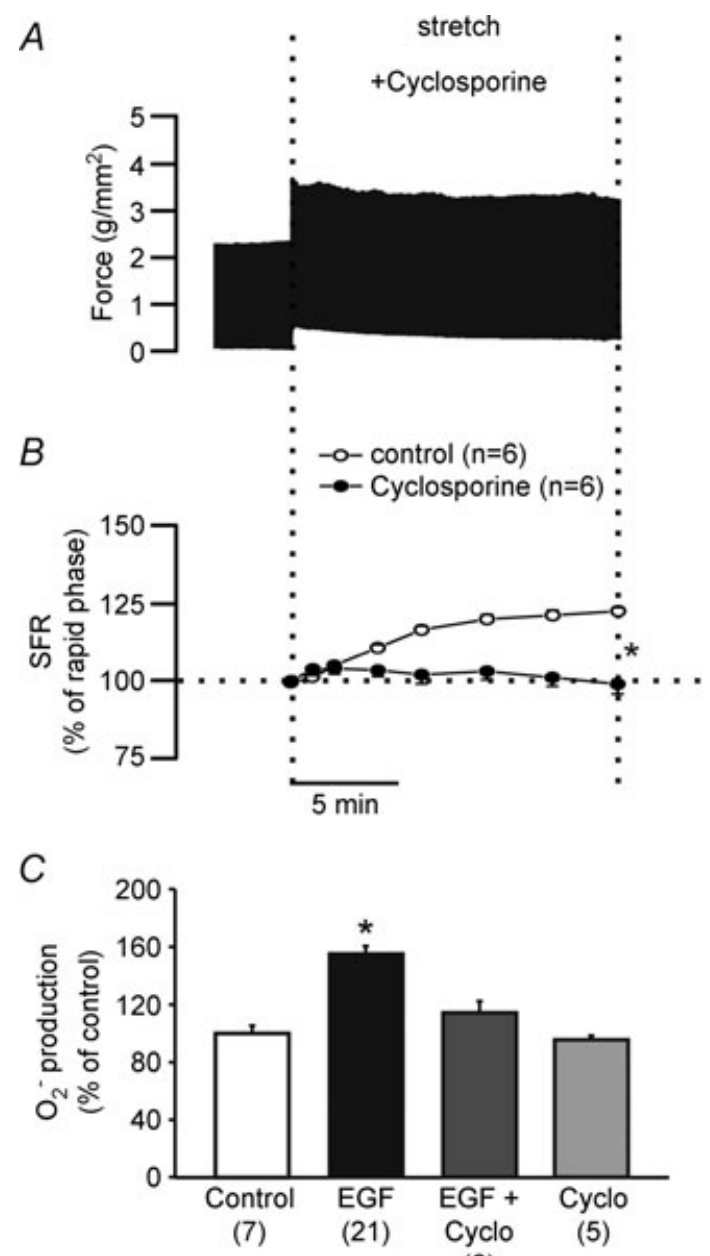

(6)

Figure 7. Role of MPTP in the development of the SFR

MPTP inhibition with cyclosporine cancelled the SFR as shown in A (representative force record) and B (averaged results expressed as a percentage of the initial rapid phase). Interestingly, cyclosporine also cancelled the increase in $\mathrm{O}_{2}{ }^{-}$production induced by $0.1 \mathrm{ng} \mathrm{ml}^{-1} \mathrm{EGF}$. These results suggest a role for MPTP in the development of the Anrep effect, probably by permitting the release of mitochondrial ROS. For the sake of comparison a control SFR already presented in Fig. $1 E$ and a control EGF-promoted $\mathrm{O}_{2}{ }^{-}$production shown in Fig. $5 \mathrm{~B}$ were also included in panels $B$ and $C$. ${ }^{*} P<0.05$ vs. control (panel $B$ ) or vs. EGF (panel C). phosphorylation was estimated by an antibody that specifically detects P-Ser703; potential changes in the phosphorylation state of other sites of the exchanger, which might contribute to the SFR, were not explored in the present work.

Our results support the notion that GPCR induced-EGFR transactivation plays a role in the chain of events that lead to NHE-1 phosphorylation and SFR development.

\section{Discussion}

In 1912, von Anrep (von Anrep, 1912) observed that, when aortic resistance was elevated, ventricular volume first increased and then declined towards its original value. An influence appeared to operate soon after the initial dilatation, which caused the heart to return toward the control volume. Rosenblueth et al. (1959) made similar observations in the right ventricle. Sarnoff et al. (1960) coined the expression 'homeometric autoregulation' to denote a mechanism that countered changes in myocardial length (Frank-Starling mechanism or heterometric autoregulation) and originated in the same myocardium.
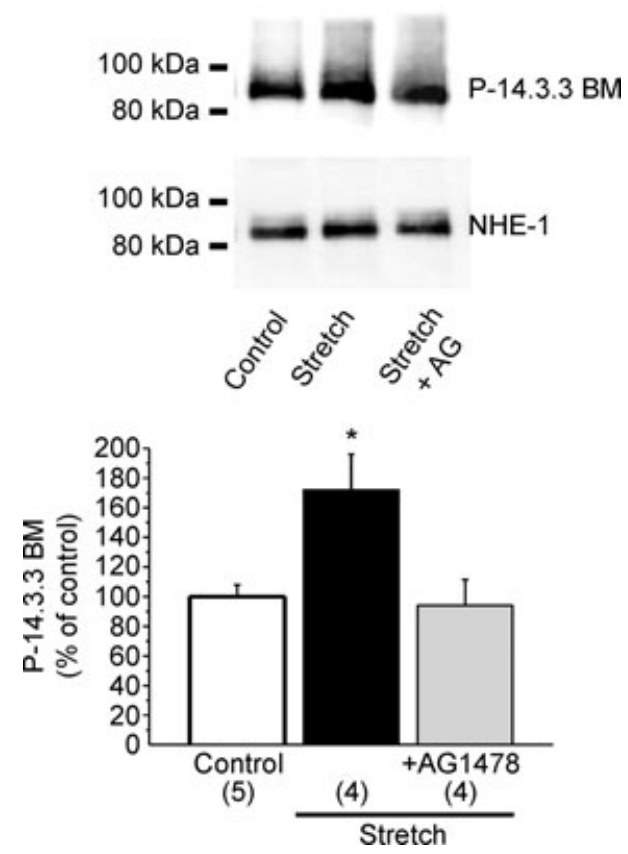

Figure 8. Stretch-induced NHE-1 phosphorylation Five minutes of stretch significantly increased NHE-1 phosphorylation at Ser703 estimated by a phospho-Ser 14-3-3 binding motif antibody. This effect was cancelled when the EGFR was blocked by AG 1478 $(A G)$. These results support a role of the EGFR transactivation in the chain of events leading to NHE-1 phosphorylation and SFR development. It is important to highlight that AG1478 alone did not modify basal NHE-1 phosphorylation ( $93 \pm 4 \%$ of control, $n=4$ ). $* P<0.05$ vs. control. 
In 1973, Parmley and Chuck reproduced this phenomenon in myocardial strips (Parmley \& Chuck, 1973). They excluded the possibility that changes in coronary blood flow could be responsible for the phenomenon (Monroe et al. 1972). Subsequently, other investigators (Allen \& Kurihara, 1982; Kentish \& Wrzosek, 1998; Alvarez et al. 1999; Calaghan \& White, 2001; Perez et al. 2001) provided further insight on the mechanisms involved in the increase in myocardial force triggered by myocardial stretching.

In our laboratory, the finding that myocardial stretch induced alkalization in cat papillary muscles bathed in bicarbonate-free medium indicated that NHE-1 was activated (Cingolani et al. 1998). We also found that NHE-1 activation was suppressed by blockade of either AT1 or ETA receptors (Cingolani et al. 2005). This led us to propose that Ang II/ET were autocrine or paracrine mediators of this phenomenon (Cingolani et al. 1998). The NHE-1-mediated increase in intracellular $\mathrm{Na}^{+}$concentration (Perez et al. 2003), the increase in action potential duration (Zhou et al. 2006), and probably a redox-sensitive phosphorylation of the cardiac $\mathrm{Na}^{+} / \mathrm{Ca}^{2+}$ exchanger (Eigel et al. 2004) appeared to be factors that contribute to the increase in contractility that follows myocardial stretch. Interestingly, in previous works, we were able to eliminate changes in $\mathrm{pH}_{\mathrm{i}}$ as a possible contributor to the SFR (Alvarez et al. 1999; Perez et al. 2001), because under physiological conditions (bicarbonate buffer), the enhanced activity of NHE-1 was not accompanied by significant changes in $\mathrm{pH}_{\mathrm{i}}$. This was due to the simultaneous Ang II activation of the $\mathrm{Na}^{+}$-independent $\mathrm{Cl}^{-} / \mathrm{HCO}_{3}{ }^{-}$anion exchanger (Alvarez et al. 2001; Cingolani et al. 2003). Several studies have shown that Ang II/ET increase ROS formation through a NADPH oxidase/mitochondrial action (Zorov et al. 2000; Seshiah et al. 2002; Cingolani et al. 2006). We recently proposed that the same signalling pathway plays a pivotal role in the genesis of the SFR to stretch (Caldiz et al. 2007) by activation of redox-sensitive kinases upstream of the NHE-1.

It has recently been established that transactivation of the EGFR is the primary mechanism that underlies GPCR agonist activation of ERK1/2 and its downstream intracellular pathways (Lemarie et al.2008). Furthermore, myocardial stretch (Anderson et al. 2004; Duquesnes et al. 2009), myocardial hypertrophy (Kagiyama et al. 2002), and ET-1 signalling (Asakura et al. 2002) have been shown to involve EGFR transactivation. The present study has provided the first evidence for the crucial role of EGFR transactivation in the development of the SFR. Based on our previous and present findings, we propose that the hypothetical signalling pathway schematised in Fig. 9 is the mechanism that underlies SFR development. We have recently proposed a crucial role of mitochondrial ROS in promoting the development of the SFR (Caldiz et al. 2007); and the present work provides conclusive evidence that EGFR transactivation is a key step in the induction of mitochondrial ROS formation.

In addition, our experimental data clearly indicated that ROS signalling takes place downstream of mitochondrial stimulation, and upstream of ERK1/2 stimulation. However, this study did not rule out any additional effects that ROS might have had on one or several other reactions in the chain of events described in Fig. 9.

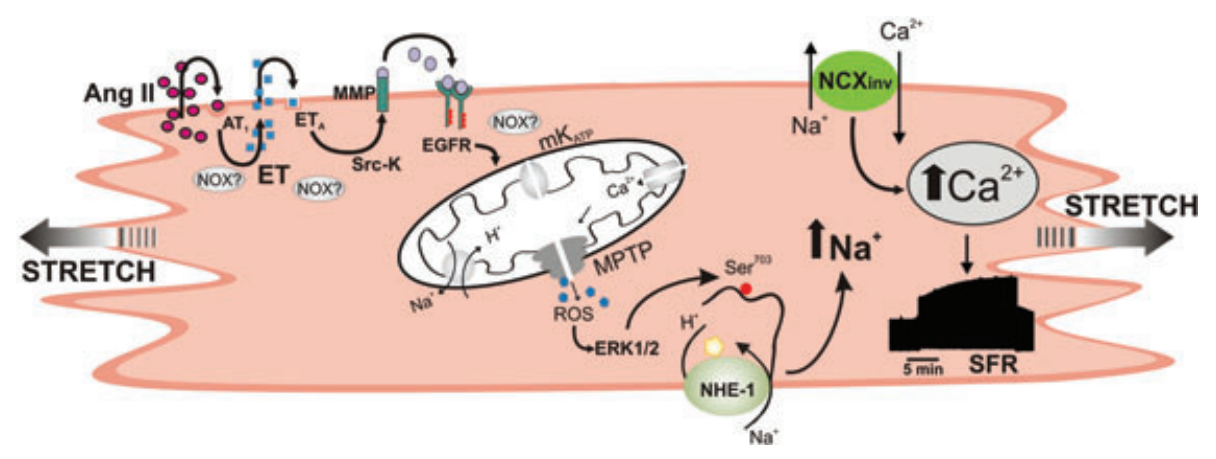

Figure 9. Scheme of the proposed mechanism leading to the SFR

Stretch and/or angiotensin II (Ang II) through AT1 receptor stimulation triggers the release/formation of endothelin (ET). NADPH oxidase-generated reactive oxygen species (ROS) probably play a significant role in this step of the signalling cascade. ET activates the $\mathrm{ET}_{\mathrm{A}}$ receptor and induces epidermal growth factor receptor (EGFR) transactivation. The communication between the $\mathrm{ET}_{\mathrm{A}}$ receptor and the EGFR involves matrix metalloprotease (MMP) activation and possibly the ROS-sensitive Src kinase. EGFR activation triggers an intracellular signalling pathway that leads to mKATP channel opening increasing ROS production and release through the mitochondrial permeability transition pore (MPTP). This causes ERK1/2 activation and NHE-1 phosphorylation at Ser703, increasing NHE-1 activity and intracellular $\mathrm{Na}^{+}$concentration that favours intracellular $\mathrm{Ca}^{2+}$ accumulation through $\mathrm{Na} \mathrm{Na}^{+} / \mathrm{Ca}^{2+}$ exchanger (NCX). The increase in ROS production can also contribute to the increase in intracellular $\mathrm{Ca}^{2+}$ through a direct stimulatory effect on the NCX (Eigel et al. 2004) and by increasing action potential duration (Zhou et al. 2006). 
For example, others have implicated ROS mediation in signalling pathways that lead to stretch-induced ET release (Anderson et al. 2004), kinase-dependent NHE-1 activation (Rothstein et al. 2002; Seshiah et al. 2002; Caldiz et al. 2007), MMP activation (Wetzker \& Bohmer, 2003), and EGFR phosphorylation by inactivation of a phosphatase (Salmeen et al. 2003; van Montfort et al. 2003). ROS were also proposed to mediate the opening of mKATP channels through a NADPH oxidase-dependent mechanism that subsequently induced mitochondrial ROS release (Brandes, 2005; Kimura et al. 2005). It is also important to highlight that NADPH oxidase is now recognized to exist in different subcellular localisations that enable the targeting of specific compartments. In this context, it was difficult to determine, with the methods used in this study, the precise steps in which ROS were critically important in the chain of events triggered by stretch. However, we presented evidence that EGFR transactivation is a crucial step in the ROS generation that leads to the Anrep effect.

\section{References}

Akram S, Teong HF, Fliegel L, Pervaiz S \& Clement MV (2006). Reactive oxygen species-mediated regulation of the $\mathrm{Na}^{+}-\mathrm{H}^{+}$ exchanger 1 gene expression connects intracellular redox status with cells' sensitivity to death triggers. Cell Death Differ 13, 628-641.

Alvarez BV, Fujinaga J \& Casey JR (2001). Molecular basis for angiotensin II-induced increase of chloride/bicarbonate exchange in the myocardium. Circ Res 89, 1246-1253.

Alvarez BV, Perez NG, Ennis IL, Camilion de Hurtado MC \& Cingolani HE (1999). Mechanisms underlying the increase in force and $\mathrm{Ca}^{2+}$ transient that follow stretch of cardiac muscle: a possible explanation of the Anrep effect. Circ Res 85, 716-722.

Allen DG \& Kurihara S (1982). The effects of muscle length on intracellular calcium transients in mammalian cardiac muscle. J Physiol 327, 79-94.

Anderson HD, Wang F \& Gardner DG (2004). Role of the epidermal growth factor receptor in signalling strain-dependent activation of the brain natriuretic peptide gene. J Biol Chem 279, 9287-9297.

Asakura M, Kitakaze M, Takashima S, Liao Y, Ishikura F, Yoshinaka T, Ohmoto H, Node K, Yoshino K, Ishiguro H, Asanuma H, Sanada S, Matsumura Y, Takeda H, Beppu S, Tada M, Hori M \& Higashiyama S (2002). Cardiac hypertrophy is inhibited by antagonism of ADAM12 processing of HB-EGF: metalloproteinase inhibitors as a new therapy. Nat Med 8, 35-40.

Brandes RP (2005). Triggering mitochondrial radical release: a new function for NADPH oxidases. Hypertension 45, 847-848.

Calaghan SC \& White E (2001). Contribution of angiotensin II, endothelin 1 and the endothelium to the slow inotropic response to stretch in ferret papillary muscle. Pflugers Arch 441, 514-520.
Caldiz CI, Garciarena CD, Dulce RA, Novaretto LP, Yeves AM, Ennis IL, Cingolani HE, Chiappe de Cingolani G \& Perez NG (2007). Mitochondrial reactive oxygen species activate the slow force response to stretch in feline myocardium. J Physiol 584, 895-905.

Cingolani HE, Alvarez BV, Ennis IL \& Camilion de Hurtado MC (1998). Stretch-induced alkalinization of feline papillary muscle: an autocrine-paracrine system. Circ Res 83, 775-780.

Cingolani HE, Chiappe GE, Ennis IL, Morgan PG, Alvarez BV, Casey JR, Dulce RA, Perez NG \& Camilion de Hurtado MC (2003). Influence of $\mathrm{Na}^{+}$-independent $\mathrm{Cl}^{-}-\mathrm{HCO}_{3}{ }^{-}$ exchange on the slow force response to myocardial stretch. Circ Res 93, 1082-1088.

Cingolani HE, Perez NG, Aiello EA \& de Hurtado MC (2005). Intracellular signalling following myocardial stretch: an autocrine/paracrine loop. Regul Pept 128, 211-220.

Cingolani HE, Villa-Abrille MC, Cornelli M, Nolly A, Ennis IL, Garciarena C, Suburo AM, Torbidoni V, Correa MV, Camilion de Hurtado MC \& Aiello EA (2006). The positive inotropic effect of angiotensin II: role of endothelin-1 and reactive oxygen species. Hypertension 47, 727-734.

Dikalov S, Griendling KK \& Harrison DG (2007). Measurement of reactive oxygen species in cardiovascular studies. Hypertension 49, 717-727.

Drummond GB (2009). Reporting ethical matters in The Journal of Physiology: standards and advice. J Physiol 587, 713-719.

Duquesnes N, Vincent F, Morel E, Lezoualc'h F \& Crozatier B (2009). The EGF receptor activates ERK but not JNK Ras-dependently in basal conditions but ERK and JNK activation pathways are predominantly Ras-independent during cardiomyocyte stretch. Int J Biochem Cell Biol 41, 1173-1181.

Eigel BN, Gursahani H \& Hadley RW (2004). ROS are required for rapid reactivation of $\mathrm{Na}^{+} / \mathrm{Ca}^{2+}$ exchanger in hypoxic reoxygenated guinea pig ventricular myocytes. Am J Physiol Heart Circ Physiol 286, H955-963.

Ennis IL, Cingolani HE, Garciarena CD, Camilión de Hurtado MC, Villa-Abrille MC, Aiello EA \& Pérez NG (2007). From Anrep's Phenomenon to myocardial hypertrophy: Role of the $\mathrm{Na}^{+} / \mathrm{H}^{+}$exchanger. Curr Cardiol Rev 3, 149-164.

Fliegel L \& Karmazyn M (2004). The cardiac Na-H exchanger: a key downstream mediator for the cellular hypertrophic effects of paracrine, autocrine and hormonal factors. Biochem Cell Biol 82, 626-635.

Haworth RS, McCann C, Snabaitis AK, Roberts NA \& Avkiran $M$ (2003). Stimulation of the plasma membrane $\mathrm{Na}^{+} / \mathrm{H}^{+}$ exchanger NHE1 by sustained intracellular acidosis. Evidence for a novel mechanism mediated by the ERK pathway. J Biol Chem 278, 31676-31684.

Ito H, Hirata Y, Adachi S, Tanaka M, Tsujino M, Koike A, Nogami A, Murumo F \& Hiroe M (1993). Endothelin-1 is an autocrine/paracrine factor in the mechanism of angiotensin II-induced hypertrophy in cultured rat cardiomyocytes. J Clin Invest 92, 398-403.

Kagiyama S, Eguchi S, Frank GD, Inagami T, Zhang YC \& Phillips MI (2002). Angiotensin II-induced cardiac hypertrophy and hypertension are attenuated by epidermal growth factor receptor antisense. Circulation 106, 909-912. 
Kentish JC \& Wrzosek A (1998). Changes in force and cytosolic $\mathrm{Ca}^{2+}$ concentration after length changes in isolated rat ventricular trabeculae. J Physiol 506, 431-444.

Kimura S, Zhang GX, Nishiyama A, Shokoji T, Yao L, Fan YY, Rahman M, Suzuki T, Maeta H \& Abe Y (2005). Role of $\mathrm{NAD}(\mathrm{P}) \mathrm{H}$ oxidase- and mitochondria-derived reactive oxygen species in cardioprotection of ischemic reperfusion injury by angiotensin II. Hypertension 45, 860-866.

Krieg T, Cui L, Qin Q, Cohen MV \& Downey JM (2004). Mitochondrial ROS generation following acetylcholine-induced EGF receptor transactivation requires metalloproteinase cleavage of proHB-EGF. J Mol Cell Cardiol 36, 435-443.

Lehoux S, Abe J, Florian JA \& Berk BC (2001). 14-3-3 Binding to $\mathrm{Na}^{+} / \mathrm{H}^{+}$exchanger isoform-1 is associated with serum-dependent activation of $\mathrm{Na}^{+} / \mathrm{H}^{+}$exchange. J Biol Chem 276, 15794-15800.

Lemarie CA, Paradis P \& Schiffrin EL (2008). New insights on signalling cascades induced by cross-talk between angiotensin II and aldosterone. J Mol Med 86, 673-678.

Leri A, Claudio PP, Li Q, Wang X, Reiss K, Wang S, Malhotra A, Kajstura J \& Anversa P (1998). Stretch-mediated release of angiotensin II induces myocyte apoptosis by activating p53 that enhances the local renin-angiotensin system and decreases the Bcl-2-to-Bax protein ratio in the cell. J Clin Invest 101, 1326-1342.

Luers C, Fialka F, Elgner A, Zhu D, Kockskamper J, von Lewinski D \& Pieske B (2005). Stretch-dependent modulation of $\left[\mathrm{Na}^{+}\right]_{\mathrm{i}},\left[\mathrm{Ca}^{2+}\right]_{\mathrm{i}}$, and $\mathrm{pHi}$ in rabbit myocardium: a mechanism for the slow force response. Cardiovasc Res 68, 454-463.

Monroe RG, Gamble WJ, LaFarge CG, Kumar AE, Stark J, Sanders GL, Phornphutkul C \& Davis M (1972). The Anrep effect reconsidered. J Clin Invest 51, 2573-2583.

Parmley WW \& Chuck L (1973). Length-dependent changes in myocardial contractile state. Am J Physiol 224, 1195-1199.

Perez NG, Caldiz CI, Chiappe de Cingolani GE \& Cingolani HE (2009). The slow force response to myocardial stretch requires transactivation of the epidermal growth factor receptor. Circulation 120, S782-S783.

Perez NG, de Hurtado MC \& Cingolani HE (2001). Reverse mode of the $\mathrm{Na}^{+}-\mathrm{Ca}^{2+}$ exchange after myocardial stretch: underlying mechanism of the slow force response. Circ Res 88, 376-382.

Perez NG, Villa-Abrille MC, Aiello EA, Dulce RA, Cingolani HE \& Camilion de Hurtado MC (2003). A low dose of angiotensin II increases inotropism through activation of reverse $\mathrm{Na}^{+} / \mathrm{Ca}^{2+}$ exchange by endothelin release. Cardiovasc Res 60, 589-597.

Rosenblueth A, Alanis J, Lopez E \& Rubio R (1959). The adaptation of ventricular muscle to different circulatory conditions. Arch Int Physiol Biochim 67, 358-373.

Rothstein EC, Byron KL, Reed RE, Fliegel L \& Lucchesi PA (2002). $\mathrm{H}_{2} \mathrm{O}_{2}$-induced $\mathrm{Ca}^{2+}$ overload in NRVM involves ERK1/2 MAP kinases: role for an NHE-1-dependent pathway. Am J Physiol Heart Circ Physiol 283, H598-605.

Sabri A, Byron KL, Samarel AM, Bell J \& Lucchesi PA (1998). Hydrogen peroxide activates mitogen-activated protein kinases and $\mathrm{Na}^{+}-\mathrm{H}^{+}$exchange in neonatal rat cardiac myocytes. Circ Res 82, 1053-1062.
Sadoshima J, Xu Y, Slayter HS \& Izumo S (1993). Autocrine release of angiotensin II mediates stretch-induced hypertrophy of cardiac myocytes in vitro. Cell 75, 977-984.

Salmeen A, Andersen JN, Myers MP, Meng TC, Hinks JA, Tonks NK \& Barford D (2003). Redox regulation of protein tyrosine phosphatase $1 \mathrm{~B}$ involves a sulphenyl-amide intermediate. Nature 423, 769-773.

Sarnoff SJ, Mitchell JH, Gilmore JP \& Remensnyder JP (1960). Homeometric autoregulation in the heart. Circ Res $\mathbf{8}$, 1077-1091.

Seshiah PN, Weber DS, Rocic P, Valppu L, Taniyama Y \& Griendling KK (2002). Angiotensin II stimulation of $\mathrm{NAD}(\mathrm{P}) \mathrm{H}$ oxidase activity: upstream mediators. Circ Res 91, 406-413.

Snabaitis AK, D’Mello R, Dashnyam S \& Avkiran M (2006). A novel role for protein phosphatase $2 \mathrm{~A}$ in receptor-mediated regulation of the cardiac sarcolemmal $\mathrm{Na}^{+} / \mathrm{H}^{+}$exchanger NHE1. J Biol Chem 281, 20252-20262.

Szokodi I, Kerkela R, Kubin AM, Sarman B, Pikkarainen S, Konyi A, Horvath IG, Papp L, Toth M, Skoumal R \& Ruskoaho H (2008). Functionally opposing roles of extracellular signal-regulated kinase $1 / 2$ and p38 mitogen-activated protein kinase in the regulation of cardiac contractility. Circulation 118, 1651-1658.

Takahashi E, Abe J, Gallis B, Aebersold R, Spring DJ, Krebs EG \& Berk BC (1999). p90(RSK) is a serum-stimulated $\mathrm{Na}^{+} / \mathrm{H}^{+}$ exchanger isoform-1 kinase. Regulatory phosphorylation of serine 703 of $\mathrm{Na}^{+} / \mathrm{H}^{+}$exchanger isoform-1. J Biol Chem 274, 20206-20214.

van Montfort RL, Congreve M, Tisi D, Carr R \& Jhoti H (2003). Oxidation state of the active-site cysteine in protein tyrosine phosphatase 1B. Nature 423, 773-777.

von Anrep G (1912). On the part played by the suprarenals in the normal vascular reactions on the body. J Physiol 45, 307-317.

Wetzker R \& Bohmer FD (2003). Transactivation joins multiple tracks to the ERK/MAPK cascade. Nat Rev Mol Cell Biol 4, 651-657.

Yamazaki T, Komuro I, Kudoh S, Zou Y, Nagai R, Aikawa R, Uozumi H \& Yazaki Y (1998). Role of ion channels and exchangers in mechanical stretch-induced cardiomyocyte hypertrophy. Circ Res 82, 430-437.

Yamazaki T, Komuro I, Kudoh S, Zou Y, Shiojima I, Hiroi Y, Mizuno T, Maemura K, Kurihara H, Aikawa R, Takano H \& Yazaki Y (1996). Endothelin-1 is involved in mechanical stress-induced cardiomyocyte hypertrophy. J Biol Chem 271, 3221-3228.

Zhai P, Galeotti J, Liu J, Holle E, Yu X, Wagner T \& Sadoshima $\mathrm{J}$ (2006). An angiotensin II type 1 receptor mutant lacking epidermal growth factor receptor transactivation does not induce angiotensin II-mediated cardiac hypertrophy. Circ Res 99, 528-536.

Zhang YH, Dingle L, Hall R \& Casadei B (2009). The role of nitric oxide and reactive oxygen species in the positive inotropic response to mechanical stretch in the mammalian myocardium. Biochim Biophys Acta 1787, 811-817.

Zhou C, Ziegler C, Birder LA, Stewart AF \& Levitan ES (2006). Angiotensin II and stretch activate NADPH oxidase to destabilize cardiac Kv4.3 channel mRNA. Circ Res 98, 1040-1047. 
Zorov DB, Filburn CR, Klotz LO, Zweier JL \& Sollott SJ (2000). Reactive oxygen species (ROS)-induced ROS release: a new phenomenon accompanying induction of the mitochondrial permeability transition in cardiac myocytes. J Exp Med 192, 1001-1014.

\section{Author contributions}

M.C.V.A., H.E.C. and N.G.P.: conception and design of the experiments, collection, analysis and interpretation of data, drafting the article and revising it critically for important intellectual content. M.B.N., M.J.C. and C.I.C.: collection, analysis and interpretation of data. I.L.E. and G.E.C.de C.: conception and design of the experiments, drafting the article and revising it critically for important intellectual content. All authors have read and approved the final version of the manuscript.

\section{Acknowledgements}

This work was supported in part by grants PICT 25475, 01031 and 78 from Agencia Nacional de Promoción Científica of Argentina to H.E.C., N.G.P. and G.E.C.de C., respectively, and PIP 1386 from CONICET to N.G.P. M.B.N. is a Fellow of Consejo Nacional de Investigaciones Científicas y Técnicas (CONICET), Argentina. M.C.V.A., I.L.E., G.E.C.de C., H.E.C. and N.G.P. are Established Investigators of CONICET, Argentina. 